\title{
Rat pups are potent reinforcers to the maternal animal: Effects of experience, parity, hormones, and dopamine function
}

\author{
A. S. FLEMING, MARC KORSMIT, and MARK DELLER \\ University of Toronto, Erindale College, Mississauga, Ontario, Canada
}

\begin{abstract}
These experiments were designed to determine whether the parity difference in robustness of a maternal experience reflects a parity difference in the reinforcing value of pups and factors affecting pup reinforcement. Postpartum and virgin animals were exposed for 15, 30, or $60 \mathrm{~min}$ to a novel environment (either a horizontally or vertically striped box) in the presence or absence of pups (or food stimuli) over a 2-, 4-, or 8-day period and then tested for their box preference in a two-choice conditioned place preference paradigm. Postpartum animals preferred the pupassociated box to the alternative box; nulliparous animals did not. The reverse pattern was found when food, rather than pups, was paired with the distinctive environment. However, if nulliparous animals were induced to become maternal either by induction procedures or by hormonal manipulation, they also preferred the pup-associated box. The effect was most robust when animals were stimulated to be maternal by hormones. Finally, pup reinforcement shares properties with other types of reinforcers in being dependent on the functional integrity of the dopamine system. Postpartum animals treated with $\operatorname{cis}(Z)$-flupentixol, a dopamine antagonist, do not develop a conditioned place preference when pups are the reinforcing stimulus. Taken together, these experiments show that pups are potent reinforcers to the maternal animal, regardless of how animals become maternal, but that the hormones of parturition may augment these effects. They show further that pup reinforcement, like food or drug reinforcement, is dopamine dependent.
\end{abstract}

Postpartum dams exhibit heightened maternal responsiveness after long periods of separation from pups if they have had the opportunity to briefly interact with them during the early postpartum period (Bridges, 1975, 1977; Cohen \& Bridges, 1981; Fleming \& Sarker, 1990; Orpen \& Fleming, 1987); as little as $1 \mathrm{~h}$ of interaction with pups is all that is required for responsiveness to be retained for up to 10 days (Malenfant, Barry, \& Fleming, 1991; Malenfant, O'Hearn, \& Fleming, 1991; Orpen \& Fleming, 1987); a longer exposure period results in considerably longer retention intervals (Fleming \& Sarker, 1990). In a recent series of experiments, we found that the maternal dam shows a more robust maternal experience effect than does the maternal virgin, even when both types of animal have been maternal for the same length of time (Fleming \& Sarker, 1990). That hormones probably mediate these learning/memory differences is suggested by the findings that exogenous hormones that mimic the pattern found at parturition also result in the long-term retention of a maternal experience, similar to what has been found for the postpartum dam (Fleming \& Sarker, 1990).

This research was supported by an N.S.E.R.C. operating grant to A.S.F. Many thanks to Lisa Cauchi for her help with the manuscript, to Jane Magnusson for her excellent lab work, to Derek van de Kooy for his helpful comments and ideas, and to John Hyttel of H. Lundbeck $\&$ Co., Denmark, for kindly providing us with cis(Z)-flupentixol. Correspondence should be addressed to A. S. Fleming, Department of Psychology, University of Toronto, Erindale College, Mississauga, ON, Canada L5L 1 C6.
What accounts for differences between maternal virgins and postpartum females? It may be that the parturitional hormones facilitate or augment maternal learning uniquely by acting on specific maternally related learning substrates; alternatively, these hormones could facilitate all types of learning, so that maternal learning is not uniquely influenced. A second possibility is that the parturitional hormones may not influence learning per se, but instead that they augment the salience, meaningfulness, or reinforcing value of the pup stimulus; and when pup value is elevated, learning is enhanced. Alternatively, the hormones may have more general effects and augment the reinforcing properties of a variety of stimuli and pups are in no way unique. The first of these hypotheses, the learning hypothesis, was addressed in our preceding paper (Fleming, Kuchera, Lee, \& Winocur, 1994), in which we reported moderate enhancement of general learning ability in postpartum animals, in comparison with virgins. However, this enhancement probably accounts for only a small proportion of the variance in maternal learning; a considerably larger role is probably played by parity differences in reinforcement value of pup stimulation. In the present study, we addressed the second reinforcement argument, and specifically tested the notions that (1) pups acquire stronger reinforcing properties for the dam than for the maternal virgin; (2) for the dam, but not for the virgin, pups are more reinforcing than food; (3) the elevated reward value of pups for the dam is hormonally mediated; and (4) the dopamine system mediates pup reinforcement. 
In the present experiments, we used the conditioned place preference paradigm to test the reinforcing properties of stimuli (Bechara \& van der Kooy,1989). In this paradigm, parous and nonparous females were separated from pups (or food) for either 0 or $23 \mathrm{~h}$ and reexposed to the pups (or food) on 1, 2, or 4 alternate days for 15 , 30 , or $60 \mathrm{~min}$ in a novel, visually distinct test chamber. On the other nonpup (food) days, the animals were exposed to a second distinct environment but without the stimuli. At the time of preference testing, the animals were permitted to move freely between the two environments, neither of which contained the unconditioned stimuli, and the time spent in each of the two environments constituted the measure of their preference and hence the strength of the reinforcing value of the pups (food).

\section{GENERAL METHOD}

\section{Subjects and Housing}

The subjects were 60 - to 90 -day-old virgin female rats born at Erindale College, University of Toronto, from a stock originally obtained from Charles Rivers Farms in Quebec. The animals were maintained on a 12:12-h light:dark cycle, with lights on at $0800 \mathrm{~h}$. Room temperature and humidity were maintained at $24.0^{\circ} \mathrm{C}$ and $40 \%$, respectively.

\begin{abstract}
Apparatus
The place preference boxes consisted of two $8.5 \times 16 \times 12$ in. white Plexiglas boxes, with either vertical or horizontal stripes made out of .75-in. electrical tape which were spaced approximately .75 in. apart. The tops of the boxes were open but were covered with .25-in. wire mesh screen during exposure and testing. All inner surfaces of the boxes were cleaned with $70 \%$ alcohol between exposures and between tests. For testing, the vertical and horizontal striped boxes were connected to one another by means of a clear Plexiglas central connector $(8.5 \times 4 \times 12$ in.). During exposure, all walls of the boxes were in place; during test, the end walls abutting the middle connector section were removed from each box so that the animal would have free access to both boxes. The position in the room of the boxes was kept constant from exposure to exposure so that any room or light cues used by the animal to make
\end{abstract} the discrimination would remain constant.

\section{Procedure}

The rats were housed individually in standard polypropylene shoebox cages $(48.3 \times 26.7 \times 15.6 \mathrm{~cm})$. The conditioning procedure involved an exposure phase and a test phase. The 4-day conditioning procedure is described below. For the 2 - and 8-day conditioning procedures, the number of exposures to each of the boxes was either reduced by one (2-day procedure) or increased by two (8day procedure) and the day of test occurred on Day 4 or Day 9 , respectively.

Exposure phase. For all deprived groups, on Day 1 postpartum pups were removed from dams for a 23 -h period. For nondeprived groups, pups were removed for only a 10-min period immediately prior to the exposures. On Days 2 and 4 postpartum, females were placed into either the vertical or the horizontal box with six 1- to 3-day-old pups for a 1-h period. The pup-paired box was the same for the 2 pup exposure days but was counterbalanced across animals within each group. On Days 3 and 5 , again after $23 \mathrm{~h}$ (or $10 \mathrm{~min}$, for nondeprived groups) of pup deprivation, the animals were placed into the non-pup-associated alternate box (either horizontal or vertical) for a 1-h period. On non-pup days, deprived females were given pups in their home cages for a 1-h period starting 1-2 $h$ after the $1-h$ conditioning. This procedure was adopted to equate the duration of pup deprivation on the pup and non-pup days, so that animals were equally "motivated" on the two types of days.

Six spot checks, at 10-min intervals, were made over the 1-h exposure period. For each spot check, the occurrence of a number of different maternal behaviors was recorded, including hovering over pups, exhibiting a crouch over pups, licking pups, sniffing pups, and sleeping. If animals were seen either hovering or crouching over pups, they were described as being maternal on that spot check. Thus, over six spot checks, a total of six indicates that animals were maternal throughout the exposure day. In addition, an overall maternal status rating was derived for each animal; this consisted of a scale ranging from 4 to 1: 4 (no hovering or crouching seen on any spot check; see Stern \& Johnson, 1990), 3 (hovering seen on at least two spot checks), 2 (hovering seen on three consecutive spot checks on I exposure day) and 1 (hovering seen on three consecutive spot checks on each of 2 exposure days).

Test phase. On Day 6, pup-deprived animals were placed into the center section of the place preference apparatus, between the two boxes, and they were allowed to move about freely in them for a 10 -min period. The frequency of entry and the time spent in each of the two boxes was continuously recorded by a computerbased event recorder. Measures of preference included (1) the percent of animals in each group spending greater than $50 \%$ of the time spent in both boxes, in the pup-paired box, and (2) the proportion of total time spent in the two boxes in the pup-paired box.

\section{EXPERIMENT 1 \\ The Development of a Conditioned Place Preference to Pups in Postpartum and Virgin Female Rats}

In the first experiment, we compared postpartum (PP) and virgin (V) animals in the development of a conditioned place preference. Factors that were varied included maternal experience, exposure duration, and deprivation status.

\section{Method}

Animals in the PP groups were mated by being placed into the cage of a sexually active male on the day of vaginal proestrus; they were left with the male overnight. Mating was confirmed by the presence of sperm or a vaginal plug on the following morning. Twenty-one days after mating (Day 1 with male), the females were transferred to large polycarbonate observation cages $(45.9 \times 45.9$ $\times 29 \mathrm{~cm}$ ). To check for parturition in PP animals, on Days 21 and 22 after mating, PP animals were checked for the pups during 15min spot checks throughout the day. In the experienced groups, pups were left with the female for 24-36 h, depending on the time of parturition. In the inexperienced groups, the pups were removed at 15-min intervals throughout parturition. The matched $\mathrm{V}$ groups were placed in large cages at the same time.

Experienced virgins were animals that had been induced to become maternal by continuous exposure to six 1- to 4-day-old foster pups. The pup induction procedure involved leaving pups with the female for $24 \mathrm{~h}$ and rotating pups with a recently fed litter every $24 \mathrm{~h}$. Pups remained with the female until the female exhibited pup retrieval and hovering or crouching. Once those behaviors were first observed, pups were left with the "maternal" female for an additional $24 \mathrm{~h}$ and then removed.

The experiment comprised three parts. In the first part, we tested the effects of conditioning duration in the PP animals; groups consisted of PP animals that were left with their pups for the first 24-36 $\mathrm{h}$ before the conditioning phase was initiated (maternally experienced). These animals experienced 2,4 , or 8 conditioning days (Groups PP/2, $n=16 ; \mathrm{PP} / 4, n=19$; and PP/8, $n=7$ ) for a 1 -h period on each exposure day or experienced 4 conditioning days but only $15 \mathrm{~min}$ or $30 \mathrm{~min}$ of exposure at each exposure time 
(Groups PP/4/15 min, $n=10 ; \mathrm{PP} / 4 / 30 \mathrm{~min}, n=11 ; \mathrm{PP} / 4 / 60 \mathrm{~min}$, $n=8$ ). Thus, animals experiencing 4 conditioning days were in a box with pups on 2 days for 60,30 , or $15 \mathrm{~min}$ on each of these days; on the intervening alternate days, the animals were in the other box without pups for 60,30 , or $15 \mathrm{~min}$. In the second part of the experiment, the effects of parity and of prior maternal experience were evaluated; PP animals were compared with virgin animals, and within each parity condition, animals were either maternally experienced or inexperienced (PP/EXP, $n=19$ and PP/INEXP, $n=11$; and V/EXP, $n=9$ and V/INEXP, $n=8$ ). V/EXP animals were given experience with pups by being induced to become maternal and given 24-36 h of additional contact with pups. In the third part of the experiment, the effects of deprivation duration were evaluated in PP animals only; thus, comparisons were made between PP animals that had been $23 \mathrm{~h}$ pup deprived before the 1-h experience in the two boxes (PP/EXP/DEP, $n=19$ ) and animals permitted to remain with pups throughout the 4-day conditioned place preference regimen, but removed from pups only $10 \mathrm{~min}$ prior to exposure trials, PP/EXP/NONDEP, $n=23$ ). All groups underwent a 4-day conditioning regimen.

\section{Results}

\section{Effects of Variations in the Duration of the}

\section{Conditioning Regime on Conditioned}

\section{Place Preference}

Exposure. On the 1st exposure day, all animals in the 2-, 4-, and 8-day groups that received $1 \mathrm{~h}$ of exposure per day were maternally responsive to the pups and hovered or crouched over them; in most cases, hovering was recorded during at least four of the six spot checks over the course of the 1-h period. In one-way analyses of variance (ANOVAs), there were no group differences in maternal status ratings (based on 1st day) or in frequency of spot checks during which maternal retrievals, licking, or hovering occurred. However, it should be kept in mind that the different groups had different amounts of exposure to pups in association with the novel environment, ranging from $1 \mathrm{~h}$ (in the 2-day group) to $4 \mathrm{~h}$ (in the 8day group). (Recall, females were only exposed to pups in association with the novel environment on alternate days of the total conditioned place preference.)

Despite differences in exposure durations among the $15-, 30-$, and $60-$ min groups, $70 \%, 80 \%$, and $88 \%$, (respectively) of these animals had maternal status ratings of 1 and 2, exhibiting a high level of hovering, on at least 1 of the 2 exposure days. Differences in maternal status ratings between groups were not significant, although, not surprisingly, the 60-min animals had higher numbers of spot checks in which hovering occurred.

Test. The 2-, 4-, or 8-day animals were said to have a preference if they spent more than $50 \%$ of the time in the two boxes, in the box previously associated with pups. As Figure 1 shows, the animals in both the 4- and the 8-day exposure groups developed a significant preference for the pup-paired box (binomial test, $p<.001$ and $p<$ .02 , respectively), whereas the animals in the 2-day condition did not. Thus, $94 \%$ of the 4-day group and $100 \%$ of the 8-day group developed a preference for the puppaired box, whereas only $56 \%$ of the 2-day group did. Group comparisons of the number of animals exhibiting a preference for the pup-paired box showed differences between the 8- and 2-day groups $\left[\chi^{2}(1)=5, p<.01\right]$ and between the 4- and 2-day groups $\left[\chi^{2}(1)=4, p<.05\right]$, but not between the 8- and 4-day groups.

One-way ANOVAs to compare groups in the proportion of total test time spent in the pup-paired box showed a significant group effect $[F(2,39)=7.2, p<.002]$, with differences occurring between the 2- and 4-day groups but not between other groups (Scheffé test). There was no significant correlation between the number of spot checks on the 1st exposure day during which maternal hovering occurred and the proportion of time spent in the pup box.

Comparisons among the 4-day groups that received exposures for 15,30 , or $60 \mathrm{~min}$ showed that $60 \%, 54 \%$, and $88 \%$ of the groups, respectively, exhibited a preference
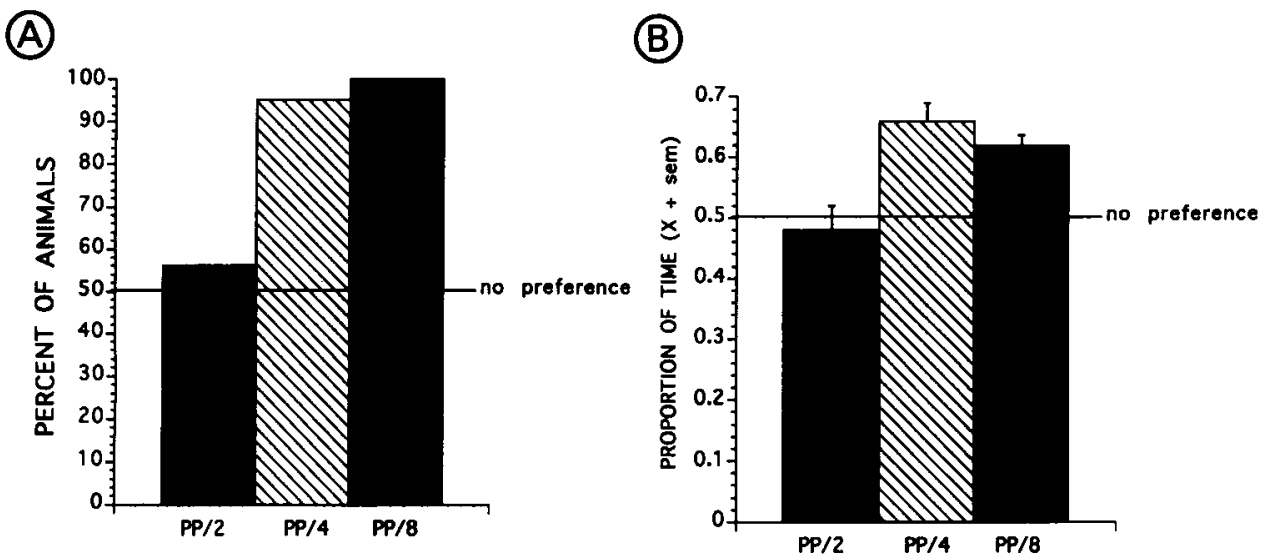

Figure 1. Animals showing a preference for the pup-paired box as a function of number of days in the exposure regimen. (A) Percent of animals preferring pup-paired box. (B) Proportion of time in pup-paired box. On each exposure day, all animals were exposed to pups for $1 \mathrm{~h}$. 
(A)

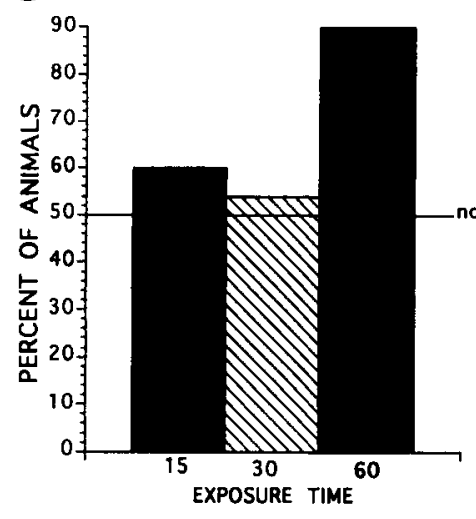

(B)

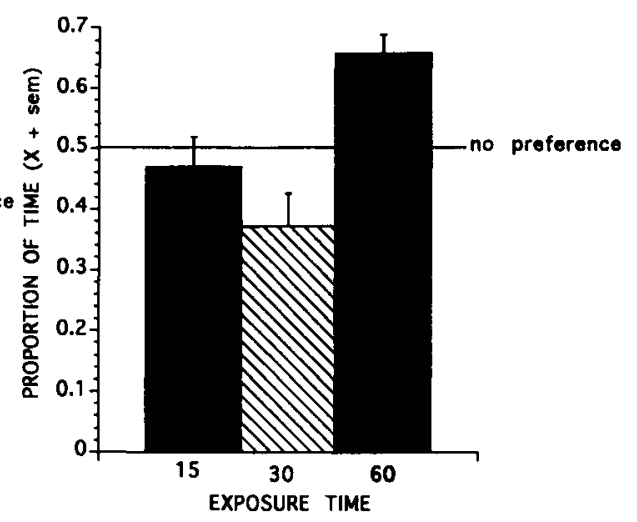

Figure 2. Animals showing a preference for the pup-paired box as a function of duration of exposure interval. (A) Percent of animals preferring pup-paired box. (B) Proportion of time in pup-paired box. Animals had a 4-day exposure regimen.

for the pup-paired box. Although these percentages were not significantly different (due to small sample sizes), binomial tests indicated that group preferences were not significant for the 15- and 30-min groups, but were significant for the 60-min group $(p<.01)$. (See Figure 2A.)

One-way ANOVAs to compare groups in the proportion of time spent in the pup-paired box showed a significant group effect $[F(2,26)=4.0, p<.01]$, with post hoc differences occurring between the 60 -min group and each of the other groups, but not between the 15- and 30min groups. (See Figure 2B.)

There were no significant correlations between the frequency of behaviors exhibited or the maternal status at exposure and the proportion of time spent in the pup box at test.

These results indicate that a single 1-h pairing is not adequate to produce a significant conditioned preference (2-day regimen), whereas with two or more 1-h pup pairings (4- and 8-day regimens), it is. Furthermore, for animals receiving two pup pairings (4-day regimens), exposure periods of 15 and $30 \mathrm{~min}$ are not adequate to produce a conditioned place preference, whereas $1 \mathrm{~h}$ is. Although there were no correlations between behavior exhibited during exposure and proportional time spent in the puppaired box during test, the more time animals spent with the pups during exposure, the more time they spent in a crouch over them. Taken together, these data indicate that the optimal conditions to reveal the strongly reinforcing effects of pups are the two 1-h pup-box pairings occurring over a 4-day period during the first 6 postpartum days, when pups are still stimulating and hormonal priming effects are still present.

\section{Effects of Prior Maternal Experience on Conditioned Place Preference}

Exposure. During the exposures both Groups PP/EXP and V/EXP exhibited some maternal behavior. However, comparisons between Groups PP/EXP and V/EXP in fre- quency of recorded maternal hoverings showed that Group $\mathrm{PP} / \mathrm{EXP}$ showed significantly higher levels $[t(26)=9.7$, $p<.0001]$. There was also a significant difference between Groups PP/INEXP and V/INEXP in the number of spot checks in which maternal hovering occurred $[t(17)=3.3, p<.004]$, the PP group again showing the highest frequency. Thus, there were also group differences in the maternal status of the two groups.

Test. As can be seen in Figure 3, 94\% and 78\% of Groups PP/EXP and V/EXP, respectively, exhibited a preference for the pup-paired box, (binomial test, $p<$ .001 and $p=.08$, one tailed, respectively); these groups did not differ from one another either in the group proportions exhibiting a preference or in proportion of time spent in the pup-paired side. Among the inexperienced groups neither PP/INEXP nor V/INEXP animals exhibited a significant preference for the pup-paired box and there were no differences between these two groups in either the proportion exhibiting a preference or the proportional time spent in the pup-paired box.

\section{Effects of Deprivation State on Conditioned Place Preference}

Exposure. During the exposure phase, both groups exhibited a high level of maternal behavior, with $90 \%$ of both groups having maternal status ratings of at least 1 or 2. There were no group differences in the frequency of spot checks in which hovering or crouching was observed.

Test. As indicated above, and as shown in Figure 4, 94\% of PP/EXP/DEPRIV animals exhibited a preference for the pup-paired box (binomial test, $p<.0001$ ); in contrast, only $70 \%$ of the PP/EXP/NONDEP animals did (binomial test, $p=.09)$. This difference was significant $\left[\chi^{2}=4.3\right.$, $d f=1, p<.03]$. Comparisons between the two groups in the proportion of time spent in the pup-paired box indicated, however, only a marginal difference on this measure $[t(40)=1.4, p=.07$, one tailed]. 
(A)

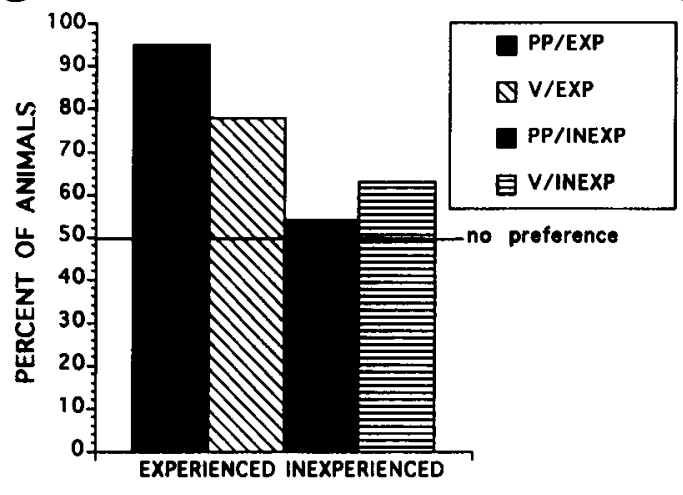

(B)

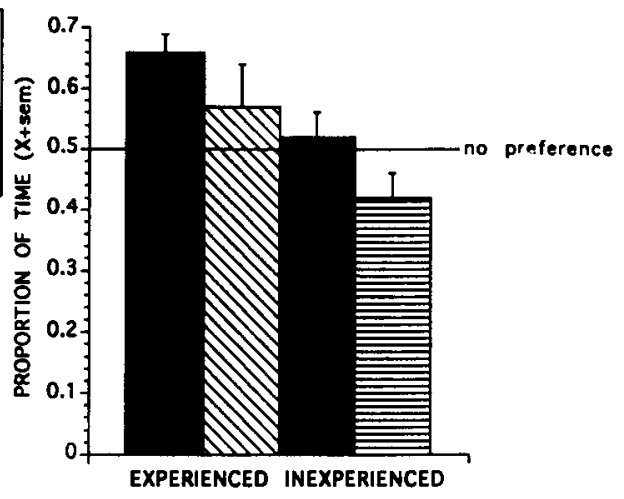

Figure 3. Animals showing a preference for the pup-paired box as a function of their parity (postpartum, PP, vs. virgin, V) and maternal experience (maternally experienced, EXP, vs. inexperienced, INEXP). (A) Percent of animals preferring pup-paired box. (B) Proportion of time in pup-paired box. A 4-day exposure regimen was used and animals were exposed to pups for $1 \mathrm{~h}$ on each exposure day.

(A)

(B)
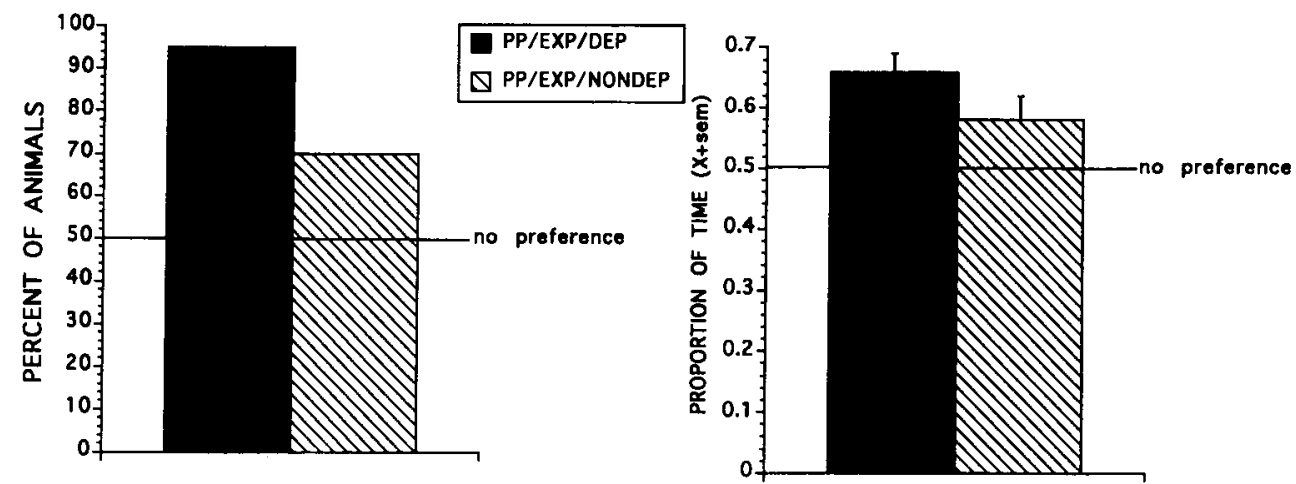

Figure 4. Animals showing a preference for the pup-paired box as a function of pup deprivation state at time of exposures. (A) Percent of animals preferring pup-paired box. (B) Proportion of time in pup-paired box. A 4-day exposure regimen was used and animals were exposed to pups for $1 \mathrm{~h}$ on each exposure day.

\section{Discussion}

These results indicate that if animals have had a maternal experience and are maternally responsive to pups in the exposure box after $24 \mathrm{~h}$ of pup deprivation, they develop a preference for the pup-associated box, regardless of their parturitional (and, hence hormonal) status at the time of the pup pairings. These data also indicate that a conditioned place preference to a pup-associated box will not occur if the dams are not pup deprived at the time of the pairings. Nondeprived dams, although maternal in the pup box, nevertheless do not develop a strong conditioned place preference.

\section{EXPERIMENT 2 \\ Conditioned Place Preference for Pup Versus Food Reinforcement in Postpartum and Virgin Animals}

The first experiment showed that pup-deprived maternal dams find pups to be more reinforcing than virgins do, even maternal virgins that are similarly pup deprived. To determine whether dams also find other stimuli to be more reinforcing or whether pups acquire particularly reinforcing properties for the new mother, in Experiment 2 we compared food-deprived virgins and new mothers in terms 
of conditioned place preference performance, with food, rather than pups, as the primary reinforcer.

\section{Method}

Five groups of animals were tested in a 4-day place preference regimen, using a 1-h exposure period, as described above. Group V/Food/No Pups $(n=18)$ were 23-h food-deprived virgins; Group PP/Food/Pups ( $n=13$ ) were Day $1 \mathrm{PP}$ animals, food deprived for $23 \mathrm{~h}$ and left with suckling pups; Group PP/Food/No pups $(n=10)$ had their pups removed at parturition and were tested after a 23-h food deprivation schedule with food. The animals in all groups underwent the same procedures as those described above, with the exception that instead of pups, six food Purina lab chow pellets were placed into the exposure box and animals were left to eat the food for a 1-h period. On non-food days, the animals were placed into an empty box for a 1-h period and given food in their home cages for a 1-h period after the exposure period.

\section{Results}

Seventy-three percent of the V/Food/No Pup animals developed a preference for the food exposure box (binomial test, $p=.04$, one tailed), whereas only $50 \%$ of Group PP/Food/No Pups did. When PP animals were left with pups and therefore experienced heightened energy expenditure, $61 \%$ (n.s.) developed a preference. Although these data suggest elevated preference among the virgins in a food paradigm, group comparisons of percent of animals showing a preference or of proportion of time spent in the food-associated box showed no significant differences.

These results suggest that PP animals do not find all stimuli to be reinforcing and suggest that the elevated reinforcing properties of pups is due to the particular salience of pups to the dam. In fact, if anything, under conditions of food deprivation, virgins find food to be reinforcing whereas mothers do not, even when the dams' energetics are being challenged by suckling young.

\section{EXPERIMENT 3 \\ Effects of Maternal Hormones on the Development of a Conditioned Place Preference in Response to Pup Stimulation}

In Experiment 3, we investigated the effects of the parturitional hormones on the development of maternal responsiveness and conditioned place preference in ovariectomized $\mathrm{V}$ rats.

\section{Method}

\section{Hormone Regimen}

Four groups of ovariectomized V females were tested. All females were ovariectomized by a midventral approach under ether anaesthesia 1 week before the initiation of hormone treatment.

On Day 1 of the hormone regimen, Groups EXP/HOR $(n=10)$ and INEXP/HOR $(n=10)$ received one silastic capsules $(5 \mathrm{~mm}$ in length) implant containing crystalline 17B-estradiol (Sigma Chemical); on Days 3-15, they received daily s.c. injections of progesterone $(5 \mathrm{mg} / \mathrm{kg}$ in $0.2 \mathrm{ml}$ of peanut oil), and on Day 15 they received a single injection of estradiol benzoate $(5 \mu \mathrm{g}$ per animal in $0.1 \mathrm{ml}$ of peanut oil). Control groups EXP/CHOL and INEXP/CHOL received one silastic capsule $(5 \mathrm{~mm})$ containing cholesterol and daily injections of $0.2 \mathrm{ml}$ of peanut oil. The silastic capsules were placed under the skin and were later removed through incisions on the upper back, under ether anesthesia.

\section{Silastic Capsule Preparation}

Lengths $(5 \mathrm{~mm})$ of Dow Corning silastic tubing, measuring $3.2 \mathrm{~mm}$ o.d. and $1.6 \mathrm{~mm}$ i.d. were packed with either crystalline 17B-estradiol (Sigma Chemical) or cholesterol. The ends of the capsules were closed with 5-mm lengths of wooden doweling, and sealed with two applications of Medical Adhesive Silicone Type A (Dow Corning). The capsules were soaked in a $0.01 \mathrm{M}$ saline buffer solution 2 days before use.

\section{Experience Condition}

On Day 16, groups were assigned to the EXP and INEXP conditions. Experienced groups were presented with six 1- to 3-dayold foster pups and left with females for $24 \mathrm{~h}$, after which they were removed, returned to their donor mothers, and replaced by another recently fed litter. The females were observed daily for the onset of maternal behavior, using maternal induction test procedures (see above). Once animals exhibited maternal retrieving on 2 consecutive days and were seen to crouch over the pups on at least 1 day, they were separated from the pups for a 24 -h period.

\section{Conditioned Place Preference Procedure}

After $24 \mathrm{~h}$ of pup deprivation, the females were placed into the 4-day CPP regimen over a 4-day period and tested on Day 5, as described above.

\section{Maternal Inductions}

\section{Results}

Comparisons among the two groups that underwent maternal induction procedures showed that $70 \%$ of the hormonally primed animals (EXP-HOR) exhibited maternal behavior, with a median latency of 5 (IQR:2,5) days, whereas none of the 10 cholesterol-treated animals (EXPCHOL) did, even after 10 continuous days of pup contact. This difference was significant (Fisher's, $p<.006$; $U=0, n_{1}=7, n_{2}=10, p<.000$ ).

\section{Exposure}

There was a significant group difference in the proportion of animals exhibiting maternal behavior during the exposure phase. Not surprisingly, $72 \%$ of the EXP-HOR group had maternal status ratings of 1 or 2 , whereas none of the EXP-CHOL group did (Fisher's, $p<.008$ ). Among the inexperienced groups, $30 \%$ and $0 \%$ of the hormone (INEXP-HOR) and cholesterol (INEXP-CHOL) groups, respectively, had maternal status ratings of 1 or 2 during the exposures. In all cases, animals that were maternal in the pup-associated boxes had also exhibited maternal behavior during the previous induction tests. Two-way ANOVAs [2 (hormone) $\times 2$ (experience)] to compare groups in the frequency of spot checks in which hovering occurred indicated (1) a significant main effect of experience $[F(2,32)=4.6, p<.04]$, with EXP groups showing higher frequencies than INEXP groups; (2) a significant main effect of hormonal status $[F(2,32)=29$, $p<.001]$, with HORM groups showing higher frequencies than CHOL groups; and (3) a significant experience $\times$ hormone interaction $[F(2,32)=9.7, p<.004]$, in which the EXP-HOR animals exhibited significantly higher frequencies than did all the other groups (by Scheffé test). 


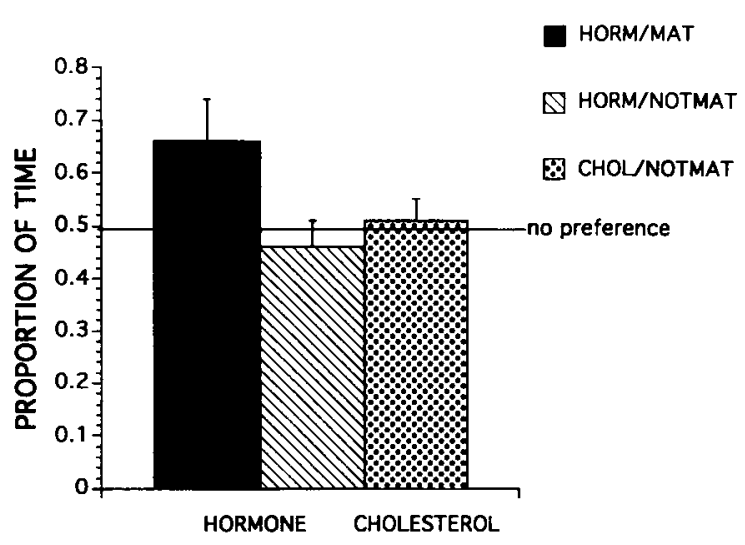

Figure 5. Animals showing a preference for the pup-paired box as a function of hormonal status and maternal status of animals; proportion of time in pup-paired box.

\section{Test}

There were no group differences in the proportion of animals exhibiting a conditioned place preference (by chi square) or in the proportion of time during the test spent in the pup-associated box (by $2 \times 2$ ANOVA). However, $68 \%$ of the hormonally treated animals that responded maternally in the pup box exhibited a significant conditioned place preference, whereas only $55 \%$ of the hormonally treated but not maternal animals did so. As is shown in Figure 5, a comparison of these two groups indicated a significant group difference in proportion of time spent in the pup-associated box [maternal, .66, vs. nonmaternal, . $46 ; t(13)=2, p<.057$ ]. It was not possible to undertake a similar comparison for the CHOL animals, since so few of them exhibited maternal behavior in the boxes. However, when all groups were combined and divided into those exhibiting maternal behavior in the pup boxes (maternal status rating of 1 or 2) versus those who did not (maternal status of 3 or 4 ), the overall difference in proportion of time spent in the pup box was significant $[t(32)=2.0, p<.05]$, with maternal animals spending proportionally more time.

\section{Discussion}

These results confirm that if virgins are administered a regimen of hormones that mimic the parturitional changes in progesterone and estradiol they can be induced to exhibit maternal behavior more rapidly than can animals receiving control substances. They show further that when paired with pups in a novel environment, maternally induced animals develop a preference for a place previously paired with pups, indicating that pups have acquired reinforcing value to them. These results also show that exposure to the hormonal regimen alone in the absence of a prior maternal experience is not adequate to sustain a preference for the pup-associated environment. However, hormonal effects are exerted indirectly by augmenting maternal responsiveness during exposure and hence the likelihood that a conditioned place preference will develop.

\section{EXPERIMENT 4 \\ Effects of cis(Z)-Flupentixol on Conditioned Place Preference in Postpartum Dams}

This last experiment was designed to determine whether the reinforcing properties of pups are mediated by the same neurochemical systems as have been found important in the food and opiate contexts (Bechera, 1991; Mackey \& van der Kooy, 1985). In a series of experiments concerned with the neurochemical mechanisms of the reinforcing effects of these other stimuli, Bechara and van der Kooy (1989) reported that a dopaminergic system mediates the rewarding effects of stimuli if an animal is in a deprived state. Thus, the dopaminergic antagonist cis $(Z)$-flupentixol (Lundbeck, Denmark) (alpha-flu) was able to block the conditioned place preference, with food or opiates as reinforcers, if animals had previously been food or drug deprived but not if they had not been previously deprived (Bechara, Harrington, Nader, \& van der Kooy, 1992).

In Experiment 4, we attempted to determine whether the dopamine system also mediates the reinforcing effects of pups for parturient females whose pups have been removed $23 \mathrm{~h}$ postpartum. We predicted that the dopamine antagonist $\operatorname{cis}(Z)$-flupentixol (Lundbeck, Denmark) should disrupt the reinforcing effects of pups and thus the development of a conditioned place preference after pup deprivation.

\section{Method}

Five groups of females were tested: a saline group $(n=15)$ and four alpha-flu groups that received $0.1 \mathrm{mg}$ alpha-flu $/ \mathrm{kg}$ of body weight $(n=15), 0.2 \mathrm{mg}$ alpha-flu $/ \mathrm{kg}$ of body weight $(n=15)$, $0.4 \mathrm{mg}$ alpha-flu $/ \mathrm{kg}$ of body weight $(n=15)$, or $0.6 \mathrm{mg}$ alphaflu $/ \mathrm{kg}$ of body weight $(n=15)$. All injections were administered i.p. in a saline vehicle.

Females gave birth (Day 0) naturally and were left with their young for 24-42 $\mathrm{h}$. The young were then removed and placed with a foster mother (Day 1). The experimental mothers were then placed in a separate room isolated from pups and pup cues.

As in the previous 4-day 60-min paradigm, on each of Days 2-5, the test subjects received an injection $2.5 \mathrm{~h}$ prior to the $1-\mathrm{h}$ exposure. On Days 2 and 4 (the pup-box days), the mother received exposure to 6 of her own pups in either the horizontal or the vertical box for $1 \mathrm{~h}$. On Days 3 and 5, the same mothers received a 1-h exposure to the other environment, not previously paired with pups, in the absence of her young. On nonpup days, the mother received a 1-h exposure to her young in her home cage 1-3 $\mathrm{h}$ after the completion of the conditioning exposure. On Day 6 postpartum, the animals were tested for their box preference, as above.

\section{Results}

The percentage of animals displaying maternal behavior $(M 1+M 2)$ during the conditioning exposures decreased as the alpha-flu dose increased (see Figure 6). One hundred percent of the subjects within both the saline group and the $0.1-\mathrm{mg} / \mathrm{kg}$ groups were maternal, whereas for successively higher concentrations of alpha-flu, $59 \%$, $46 \%$, and $37 \%$ of the subjects within the $0.2-, 0.4-$, and $0.6-\mathrm{mg} / \mathrm{kg}$ groups, respectively, attained $M 1$ or $M 2$ ratings during exposures. In fact, there was a significant dif- 
(A)

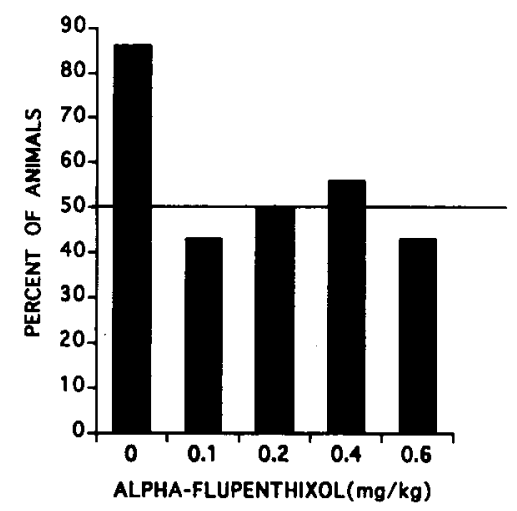

(B)

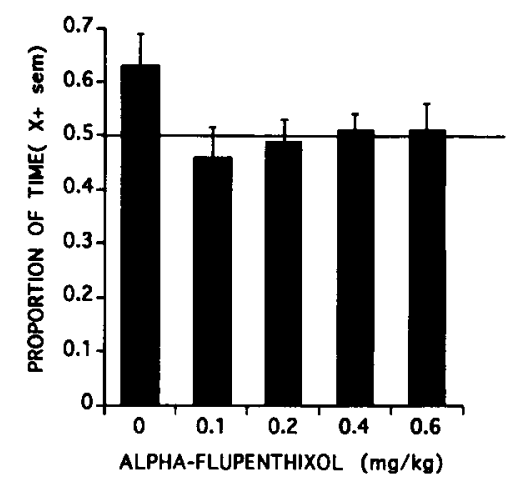

Figure 6. Animals showing a preference for the pup-paired box as a function of concentration of $c i s(Z)$-flupentixol. (A) Percent of animals preferring pup-paired box. (B) Proportion of time in pup-paired box.

ference in maternal ratings across the groups $[F(4,75)=$ $3.6, p<.01]$, which reflects a difference between both the saline and the $0.1-\mathrm{mg} / \mathrm{kg}$ group and each of the higher dose groups.

As shown in Figure 6, a significant proportion of the saline group preferred the pup-paired box (binomial test, $p<.01$ ). However, no significant preferences were found for the 4 alpha-flu groups. Consistent with these results is that there were no differences among the four alpha-flu groups in proportion of times that they were in the pup box. However, there was a sharp decline in the relative time spent in the pup-paired box between the saline and the $0.1-\mathrm{mg} / \mathrm{kg}$ alpha-flu group; the saline animals differed significantly from all the alpha-flu groups combined $[t(61)=2.8, p<.007]$ as well as from each of the individual alpha groups. To determine whether preference for the pup-paired box during test was related to the extent or intensity of maternal responding during the exposure phase, two analyses were undertaken. The first correlational analyses showed no significant relation between the number or the frequency of maternal behaviors during exposure and either the relative time or the proportion of total time in the pup-paired box during test. The second, an analysis of covariance, compared the saline group and a combined group of alpha-flu animals (with Groups $0.1 \mathrm{mg}$ to $0.6 \mathrm{mg} / \mathrm{kg}$, combined) in relative time spent in the pup box and included as the covariate either maternal status or frequency of maternal behavior during exposure. This analysis showed that the significant group difference in preference performance during test was retained with inclusion of the covariate and, therefore, was not due to group differences in maternal status during exposure $[F(1,60)=8.8, p<.004]$.

\section{Discussion}

These results indicate that the dopamine antagonist $\operatorname{cis}(Z)$-flupentixol can block the development of a conditioned place preference with pups used as a reinforcer if dams are in a pup-deprived state. Saline-injected subjects showed a strong preference for the pup-paired box during testing, whereas none of the alpha-flu groups did. These findings suggest that even the lowest concentrations of alpha-flu, at 0.1 to $0.2 \mathrm{mg} / \mathrm{kg}$, were adequate to totally block the formation of a conditioned place preference in postpartum dams, when pups were used as conditioning stimuli. It is possible that an alpha-flu concentration of less than $0.1 \mathrm{mg} / \mathrm{kg}$ would have produced less complete blocking effects, revealing a dose-response relation, and that all the alpha-flu concentrations used were, in fact, too high. However, it should be noted that in Bechara's experiments (Bechara, 1991; Bechara et al., 1992), in which food or opiates constituted the conditioning stimuli, the effective blocking concentrations were 8-10 times higher-in the $0.8-1.0 \mathrm{mg} / \mathrm{kg}$ range. Although we never administered these higher concentrations, the highest $0.6-\mathrm{mg} / \mathrm{kg}$ concentration that we did use produced serious motoric immobility, making meaningful interpretation of its blocking effects difficult. Although the alpha-flu groups exhibited less maternal behavior during exposure than did the saline animals, both the correlational and the covariance analyses showed that performance differences during exposure do not explain performance differences during the test. Thus, alpha-flu groups that exhibited strong maternal behavior during the exposures nevertheless did not behave as though pups were highly reinforcing, as is indicated by the absence of a conditioned place preference. This was particularly apparent in the $0.1-\mathrm{mg} / \mathrm{kg}$ group, where animals that were highly maternal during exposure did not show conditioned place preferences.

\section{GENERAL DISCUSSION}

Taken together, these studies show that the new mother finds pups to be highly reinforcing, whereas virgins with the same amount of exposure with pups do not. However, this parity difference in the reinforcing properties of pups 
seems to depend primarily on differences between the two kinds of animal in their motivation to respond maternally to pups. Animals that are induced to become maternal prior to the place preference task, whether through the action of hormones or by sensitization procedures, are more likely to find pups reinforcing than are animals that are not maternally responsive prior to test. Thus, maternally experienced groups exhibited a conditioned preference for the pup-associated box, whereas maternally inexperienced groups did not. Animals that were maternal prior to the exposure periods also tended to express maternal behavior in the exposure boxes. It is, therefore, not surprising that there was also a relation between behavior in the exposure boxes and preference performance at test. However, being maternal during the exposure phase may be a necessary, but not a sufficient, condition for the development of a preference for a place previously associated with rat pups. As indicated above, experienced PP animals that were maternal in the pup box were also likely to develop a conditioned place preference, whereas inexperienced PP animals that were not maternal in the pup box did not; similarly, $V$ animals that exhibited maternal behavior in the pup box developed a conditioned place preference, whereas those that did not exhibit maternal responding did not. However, for animals that exhibited maternal behavior in the pup box, there seems to be little relation between the intensity of maternal responding during exposure and the development of a conditioned place preference. In fact, PP animals not deprived of pups prior to exposure showed robust maternal behavior in the exposure boxes but no preference for the pup-associated box at test.

The findings from the first experiment that one 1-h pup-box pairing or two 30-min pup-box pairings are not adequate to sustain a conditioned place preference, although the maternal behavior exhibited during that briefer exposure suggests that for maternal behavior to be exhibited initially pups either do not need to be reinforcing or do not need to be as reinforcing to sustain maternal responding as they do to sustain a conditioned place preference. Given the importance of the parturitional hormones for the rapid initiation of maternal behavior, it is entirely possible that pups need not be particularly reinforcing initially but that with experience they acquire stronger reinforcing properties and hormones and are then able to maintain a high level of maternal responsiveness past the period when the parturitional hormones are exerting effects.

The results from Experiments 1 and 2 also indicate that the parturitional hormones, separate from their effects on maternal behavior, do not substantially augment pup reinforcement. Neither the inexperienced PP animals nor the inexperienced hormonally primed virgins developed a conditioned place preference simply as a result of their hormonal status; however, once these hormones had activated maternal behavior, pups acquired reinforcing value. In fact, a comparison of place preference performance between the experienced V and PP groups sug- gests a marginally more robust preference among the animals experiencing pups within the context of hormonal priming, and hence the parturitional hormones may indeed somewhat elevate pups hedonic status.

These experiments also show that food as a reinforcer may be a more powerful stimulus to the food-deprived virgin than to the food-deprived dam, whereas the reverse is the case for the maternal animal. Clearly, PP animals do not find all motivational stimuli to be highly reinforcing; stimulus salience depends on the animal's reproductive state.

Finally, our data indicate that pup reinforcement is mediated by the same dopaminergic mechanisms that mediate food reinforcement or drug reinforcement (Bechara, 1991; Hiroi \& White, 1990, 1991). The fact that with increasing concentrations of the dopamine antagonist there was a decrease in maternal responding probably does not reflect dopaminergic involvement in maternal motivation; rather, the disruptive effects at higher concentrations of alpha-flu are probably due to motoric disruptions. The dopaminergic neurons that are involved in the development of a deprivation-based conditioned place preference and whose effects may be blocked by alpha-flu are found primarily in the mesolimbic VTA-nucleus accumbens pathway (Hiroi \& White, 1990, 1991). In contrast, brain regions that are involved in normal maternal behavior are the medial preoptic area, the medial and cortical nuclei of the amygdala, portions of the olfactory system, the habenula, and the VTA and brainstem projections (Numan, 1988). The difference in brain structures that mediate maternal and conditioned place preference behaviors suggests that the dopaminergic antagonist alpha-flu probably does not have a direct effect on the motivation to express maternal behaviors such as hovering over, crouching, or pup licking, although recent evidence suggests that pup retrieval may be under mesolimbic dopaminergic control (Hansen, Harthon, Wallin, Löfberg, \& Svensson, 1991a, 1991b).

How maternal responsiveness during exposure functions to augment the reinforcing properties of pups in situations where they do so is not clear. We have some evidence to suggest that in order for pups to become strongly reinforcing, the dams must experience somatosensory input during their interactions. Preliminary evidence from our lab indicates that if maternally active dams are prevented from experiencing tactile input through either the perioral snout region or the ventrum (by application of a local anaesthetic), they exhibit maternal crouching and hovering over pups during exposure but they do not develop a conditioned place preference. Moreover, if dams are exposed to pups in a wire mesh cage within the pup box, so that they cannot lick or touch them but can see, hear, and smell them, they similarly do not develop a conditioned preference for the pup-associated box (Fleming, unpublished observations). These data are consistent with our previous findings that for a maternal experience to be acquired and retained, dams must experience somatosensory input from both the perioral and ventral-trunk regions 
(Morgan, Fleming, \& Stern, 1992; see also Stern \& Johnson, 1990). Simple exposure to the smell, sight, and olfactory characteristics of pups is not adequate (Orpen \& Fleming, 1987).

Finally, the findings that with maternal experience pups become highly reinforcing to the dam provides an efficient mechanism to ensure that the dam remains nurturant and attentive to her pups during the postpartum period when maternal hormones are no longer present.

\section{REFERENCES}

Bechara, A. (1991). The neurobiology of motivation: Double dissociation of motivational effects under deprivation versus nondeprivation conditions. Unpublished doctoral dissertation, University of Toronto.

Bechara, A., Harrington, F., Nader, K., \& Van der Kooy, D. (1992). Neurobiology of motivation: Double dissociation of two motivational mechanisms mediating opiate reward in drug-naive versus drug-dependent animals. Behavioral Neuroscience, 106, 798-807.

Bechara, A., \& VAN DER KoOY, D. (1989). The tegmental pedunculopontine nucleus: A brain-stem output of the limbic system critical for the conditioned place preferences produced by morphine and amphetamine. Journal of Neuroscience, 9, 3400-3409.

BRIDGES, R. S. (1975). Long-term effects of pregnancy and parturition upon maternal responsiveness in the rat. Physiology \& Behavior, 14, 245-249.

BRIDGES, R. S. (1977). Parturition: Its role in the long term retention of maternal behavior in the rat. Physiology \& Behavior, 18, 487-490.

COHEN, J., \& BridGeS, R. S. (1981). Retention of maternal behavior in nulliparous and primiparous rats: Effects of duration of previous maternal experience. Journal of Comparative \& Physiological Psychology, 95, 450-459.

Fleming, A. S., Kuchera, C., Lee, A., \& Winocur, G. (1994). Olfactory-based social learning varies as a function of parity in female rats. Psychobiology, 22, 37-43.

Fleming, A. S., \& SARKER, J. (1990). Experience-hormone interactions and maternal behavior in rats. Physiology \& Behavior, 47, 1165-1173.

Hansen, S., Harthon, C., Wallin, E., Löfberg, L., \& Svensson, K. (1991a). The effects of 6-OHDA-induced dopamine depletions in the ventral or dorsal striatum on maternal and sexual behavior in the female rat. Pharmacology, Biochemistry \& Behavior, 39, 71-77.
Hansen, S., Harthon, C., Wallin, E., Löfberg, L., \& Svensson, K. (1991b). Mesotelencephalic dopamine system and reproductive behavior in the female rat: Effects of ventral tegmental 6hydroxydopamine lesions on maternal and sexual responsiveness. Behavioral Neuroscience, 105, 588-598.

Hirol, N., \& WhITE, N. M. (1990). The reserpine-sensitive dopamine pool mediates $(+)$-amphetamine-conditioned reward in the place preference paradigm. Brain Research, 510, 33-42.

Hirol, N., \& WhITE, N. M. (1991). The amphetamine conditioned place preference: Differential involvement of dopamine receptor subtypes and two dopaminergic terminal areas. Brain Research, 552, 141-152.

MACKEY, W. B., \& VAN DER KOOY, D. (1985). Neuroleptics block the positive reinforcing effects of amphetamine but not of morphine as measured by place conditioning. Pharmacology, Biochemistry \& Behavior, 22, 101-106.

Malenfant, S. A., Barry, M., \& Fleming, A. S. (1991). Effects of cycloheximide on the retention of olfactory learning and maternal experience effects in postpartum rats. Physiology \& Behavior, 49, 289-294.

Malenfant, S. A., O’Hearn, S., \& Fleming, A. S. (1991). MK801, an NMDA antagonist, blocks acquisition of a spatial task but does not block maternal experience effects. Physiology \& Behavior, 49, 1129-1137.

Morgan, H. D., Fleming, A. S., \& Stern, J. M. (1992). Somatosensory control of the onset and retention of maternal responsiveness in primiparous Sprague-Dawley rats. Physiology \& Behavior, 51, 549-555.

Numan, M. (1988). Maternal behavior. In E. Knobil \& J. Neill (Eds.), Physiology of reproduction (Vol. 2, pp. 1569-1646). New York: Raven Press.

OrPen, B. G., \& Fleming, A. S. (1987). Experience with pups sustains maternal responding in postpartum rats. Physiology \& Behavior, 40, 47-54.

STERn, J. M., \& Johnson, S. K. (1990). Ventral somatosensory determinants of nursing behavior in Norway rats: I. Effects of variations in the quality and quantity of pup stimuli. Physiology \& Behavior, 47, 993-1011.

(Manuscript received March 18, 1993; revision accepted for publication August 19, 1993.) 